\title{
Spectrum Bonding and Aggregation With Guard-band Awareness in Cognitive Radio Networks
}

\author{
Haythem Bany Salameh \\ Dept. of Telecommunication Eng. \\ Yarmouk University, Jordan
}

\author{
Marwan Krunz \\ Dept. of Electrical and Computer Eng. \\ University of Arizona, USA
}

\author{
David Manzi \\ Raytheon Corporation \\ Arizona, USA
}

\begin{abstract}
Spectrum access/sharing algorithms for Dynamic spectrum access (DSA) networks are often designed without accounting for adjacent-channel interference. In practice, guard bands are needed to prevent such interference. Introducing guard bands naturally constrains the effective use of the spectrum. In this work, we investigate the problem of assigning channels/powers to opportunistic transmissions, while accounting for such a constraint. Specifically, we propose a novel guard-band-aware channel assignment scheme for DSA systems. Our scheme reduces the number of required guard channels for a given transmission by exploiting the benefit of utilizing adjacent channels and considering already reserved guard channels. We analytically formulate the channel access problem as a joint power control and channel assignment optimization problem, with the objective of minimizing the required spectrum resource for a given $\mathrm{CR}$ transmission. We show that the optimization problem is a binary linear program (BLP), which is, in general, NP-hard. Accordingly, we present a near-optimal solution based on sequential fixing, where the binary variables are determined iteratively by solving a sequence of linear programs. Based on the proposed channel assignment algorithm, we develop an operational MAC protocol that enables DSA users to dynamically utilize the spectrum. The proposed protocol realizes our channel assignment algorithm in a distributed manner while relying only on information provided by the two communicating users. Simulation results are provided, which verify the effectiveness of our protocol and demonstrate the significant gain achieved through guard-band-aware channel assignment.
\end{abstract}

Index Terms-Opportunistic access, guardband-awareness, adjacentchannel interference, discontinuous OFDM.

\section{INTRODUCTION}

Studies conducted by the FCC and other agencies challenged for the first time the common belief of spectrum scarcity by indicating that at any given time and in any geographic locality, less than $10 \%$ of the available spectrum is utilized [1], [2]. To exploit the highly underutilized portions of the spectrum (a.k.a, white spaces, spectrum holes, etc.), the FCC advocates the need for a new generation of smart, programmable radios that are capable of spectrum sensing, environment learning, and opportunistic spectrum access. These so-called cognitive radios (CRs) have recently been the focus of extensive research. They promise to support opportunistic spectrum access without affecting primary radio (PR) users. CR users that co-exist with PR users should frequently sense their operating channels for active PR signals to discover spectrum opportunities, and should vacate these channels if a PR signal is detected. Given the available spectrum opportunities at different $C R$ users, a crucial challenge in this domain is how nodes in a CR network (CRN) can cooperate to access the spectrum in order to efficiently utilize those opportunities while improving the overall network throughput.

\subsection{Motivation}

Various channel assignment algorithms for CRNs have been proposed in the literature (e.g., [3]-[7]). Most

An bridge version of this paper was presented at the IEEE GLOBECOM 2011 Conference.

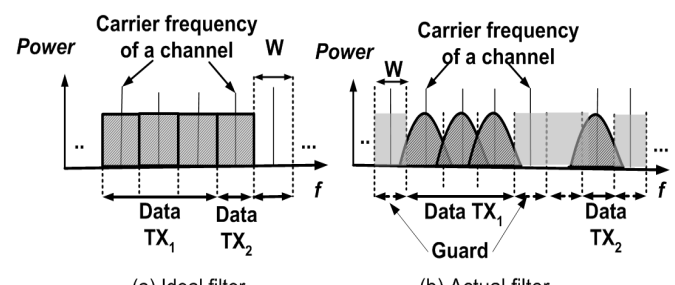

Fig. 1. Ideal vs. actual transmission filters.

of them were designed without considering adjacentchannel interference $(\mathrm{ACI})^{1}$, thus requiring ideal (rectangular) transmission filters (see Fig. 1(a)). In practice, however, spectrum spill-over is common during signal filtering. To mitigate ACI and protect neighboring PR/CR receptions, frequency separation (unused portion of spectrum) between adjacent channels is needed. Such separation is referred to as a guard band. The imposition of guard bands adds a constraint on the effective use of the spectrum. Therefore, when assigning channels/powers to CR users, it is necessary to consider the guard-band issue to improve spectrum utilization. Note that guard bands are not needed between contiguous channels that are assigned to the same transmission (we refer to a contiguously assigned set of channels as a frequency block). For every frequency block, one guard channel on each side of the block is needed (e.g., in Fig. 1(b), one frequency block of 3 channels is assigned to transmission 1, which requires 2 guard channels).

Another aspect of previously proposed channel assignment mechanisms is that they are typically based on selecting the "best" channel, or set of channels, for a given

1. ACI is caused by extraneous power received from a transmission operating on an adjacent channel. 
transmission (e.g., [8]). We refer to this approach as the greedy approach. When the greedy approach is employed in a CRN, the number of required guard channels may be quite high, resulting in a high blocking probability for CR transmissions and poor network throughput. To illustrate, consider a transmission that requires $m$ data channels. Assume that the best $m$ channels are all noncontiguous, and at least one guard channel is available on each side of each channel. According to the greedy approach, the total number of required channels (dataplus-guard) is $m+2 m=3 m$. In general, if the $m$ data channels are selected from $k$ non-contiguous frequency blocks, then the required number of channels is $m+2 k$. Hence, an efficient channel assignment algorithm should try to minimize $k$ (ideally, $k=1$ ), as illustrated in Fig. 2 .

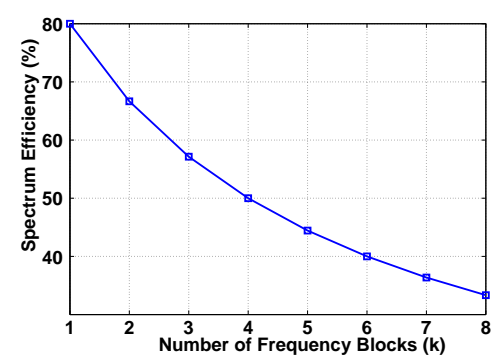

Fig. 2. Number of required channels vs. number of blocks ( $m=$ 8).

\subsection{Contributions}

In this work, we develop a distributed power-controlled contention-based MAC protocol for enhancing the throughput of a multi-channel CRN under the realistic assumption of non-ideal filters (i.e., guard bands are needed). The proposed protocol employs an intelligent guard-band-aware channel assignment mechanism. It reduces the number of required guard channels for a given transmission by minimizing the number of noncontiguous "frequency blocks" assigned to this transmission. We propose two variants of the guard-bandaware channel assignment mechanism. The first variant is suitable for CRNs with a transmission technology that does not allow two neighboring $C R$ transmissions to share the same guard channel (no guard-band reuse), while the other variant allows for guard-band reuse. Fig. 3 illustrates channel assignment with and without guardband reuse. Our MAC protocol has several attractive features. First, it does not require a central server for frequency planning, and hence it can efficiently deal with mobility. Second, it does not make any assumptions about the activities of the underlying networks. Third, it does not require interaction with PRNs, and can be adapted to existing multi-channel systems with little extra processing overhead. Finally, it considers channel dynamics and PR activity. To the best of our knowledge, it is the first protocol to account for the guard-band overhead in the channel assignment process.

We note that the impact of ACI in CRNs was previously investigated in [9]. Specifically, the authors proposed a centralized solution for adaptive guard-band configuration, called Ganache. Ganache exploits the benefit of dynamic guard-band configuration to minimize ACI. It requires a central server for frequency planning, and is oriented towards static users with similar OFDM configurations (e.g., limited node mobility). The treatment in [9] did not consider channel aggregation and did not deal with the channel assignment problem.

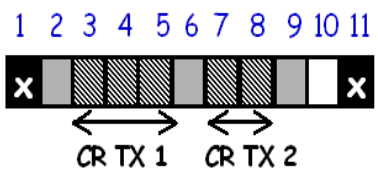

(a) With guard-band reuse

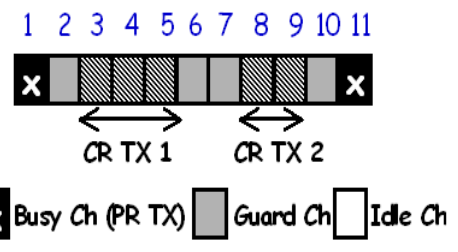

(b) Without guard-band reuse
Fig. 3. Channel assignment with/without guard-band reuse. In part (a)/(b), transmission 2 can/cannot reuse guard channel 6 , which was assigned to transmission 1.

\subsection{Organization}

The rest of the paper is organized as follows. In Section 2, we describe the system model, state the main design constraints, and formulate the channel/power assignment optimization problem. Section 3 introduces our proposed guard-band-aware channel assignment scheme. In Section 4, we integrate this scheme into a MAC protocol. Simulation results and discussion are presented in Section 5. Finally, Section 6 gives concluding remarks.

\section{Models and Problem Formulation}

\subsection{Network Model}

We consider an ad hoc CRN that coexists geographically with $L$ different PR networks (PRNs). For the $i$ th PRN, its available bandwidth $\left(B_{i}\right)$ is divided into $C_{i}$ adjacent but non-overlapping frequency channels, each of Fourier bandwidth $W$ (in $\mathrm{Hz})^{2}$. Let $M$ denote the total number of channels in the network; $M=\sum_{i=1}^{L} C_{i}$. Let $\mathcal{M}$ be the set of channels. CR users continuously scan the spectrum, identifying potential spectrum holes (idle PR channels) and opportunistically accessing them. We assume that the data rate of an idle channel is proportional to the channel bandwidth. Accordingly, a bandwidth model that delivers 1 bit per $\mathrm{Hz}$ is considered if the received SINR is greater than a given threshold $\left(\mu^{*}\right)$ [10]. Formally, for an idle channel $i \in \mathcal{M}$, its transmission rate $\left(R_{i}\right)$ is obtained according to the following rate-SINR relationship:

$$
R_{i}= \begin{cases}W \mathrm{Mbps}, & \text { if } \operatorname{SINR}^{(i)} \geq \mu^{*} \\ 0, & \text { otherwise. }\end{cases}
$$

where SINR ${ }^{(i)}$ denotes the received SINR over channel $i$. Our setup assumes a single (basic) transmission rate per

2. Without loss of generality, we assume that the spectrum is grouped into equal bandwidth frequency channels. 
channel. Extending the treatment to a multi-rate system is possible. However, because power control is already being used in our scheme, there may not be a great benefit in adapting both the transmission rate and transmission power at the same time, especially in view of our primary objective (i.e., minimizing guard-band overhead). Joint power/rate adaptation in the context of a multi-rate system is more relevant if the problem is formulated as a throughput-maximization problem subject to constraints.

A CR transmission may proceed over multiple contiguous ("bonded") or non-contiguous ("aggregated") idle channels, depending on the spectrum opportunities. This synchronized multi-channel transmission capability can be realized using frequency division multiplexing (FDM), or discontinuous orthogonal frequency division multiplexing (D-OFDM) [4], [11], [12].

We note that a number of experimental studies were previously conducted to highlight the benefits of channel bonding and aggregation in the context of CRNs (e.g., [13]-[15]]). In [13] the authors experimentally investigated the impact of using variable channel width (i.e., channel bonding) on the performance of an IEEE 802.11based network. They showed that channel bonding can significantly improve the network performance in terms of throughput, range, and power consumption. However, their treatment did not consider channel aggregation (i.e., the combining of non-contiguous portion of the spectrum to form one channel). In addition, they only considered single-hop small-network scenarios. In [14], the authors used a Markov model to assess the throughput of an adhoc opportunistic spectrum access network with channel bonding. They showed that channel bonding, in general, has great potential to improve network performance. The level of improvement heavily depends on network size and number of available channels. In addition, the results revealed that significant performance improvement can be achieved by adaptively changing the number of bonded channels according to network conditions. However, the developed model did not consider the guard band issue. The authors in [15] investigated the feasibility of opportunistic spectrum access while considering strict limit on the disruption rate at PR users. The authors showed that traditional conservative spectrum access policies cannot provide reliable communications to CR users. Accordingly, they proposed the use of "channel bundling", whereby CR users can have reliable communications by combining multiple, randomly selected, unreliable channels. None of the aforementioned papers incorporate a guard band constraint in their design.

\subsubsection{FDM-based CRNs}

In this case, each CR user is equipped with $n_{t}$ halfduplex radio transceivers, $1 \leq n_{t} \leq M$, which can be used simultaneously. The $C R$ user can transmit over an arbitrary segment of the available bandwidth by using tunable raised-cosine pulse filters with one transceiver per frequency block. When a raised-cosine filter is used, the required number of guard channels depends on the number of channels in a frequency block and the rolloff factor of the raised-cosine filter $(\beta)$. This $\beta$ is a measure ${ }^{3}$ of the excess bandwidth of the filter. Formally, for a CR transmission that uses a frequency block of $m$ adjacent channels, the excess bandwidth on each side of the frequency block is $\Delta f=m W \frac{\beta}{2}$. Thus, a necessary and sufficient condition to mitigate ACI using only one guard channel of bandwidth $W$ on each side of a frequency block is $\Delta f \leq W$, implying $m \leq \frac{2}{\beta}$. For practical values of $m, \beta$, and $W$, the above condition often holds. For example, with $\beta=0.1$ and $W=3 \mathrm{MHz}, m \leq 20$ channels (i.e., a data rate of up to $60 \mathrm{Mbps}$ ). Accordingly, it is reasonable to assume that a guardband of bandwidth $W$ on each side of a frequency block is sufficient to protect the reception over that block and avoid harmful interference to neighboring transmissions. This means that two guard channels are needed to separate two frequency blocks assigned to neighboring transmissions. This represents the case where a guard channel that is reserved for a CR transmission cannot be reused (shared) by another CR transmission (Fig. 3(b)).

\subsubsection{D-OFDM-based CRNs}

Under D-OFDM, a CR transmission can simultaneously proceed over multiple channels (contiguous or noncontiguous) using a single half-duplex radio, where each channel consists of a distinct block of the same number of contiguous sub-carriers [4], [11]. In essence, this capability can be achieved by assigning zero power to the sub-carriers of non-assigned/busy channels. For a given CR transmission and a set of assigned channels, all subcarries belonging to the selected channels will be used for that transmission [4], [11]. It has been shown that the nearest sub-carriers of a neighboring frequency block that is assigned to another transmission are the main source of interference to any demodulated sub-carrier [16], [17]. Therefore, to prevent ACI, it is sufficient to assign one guard channel between any two frequency blocks that are allocated to two different co-located CR transmissions, irrespective of the size of their frequency blocks [16], [17]. This represents the case win Fig. 3(a).

The available channel set for CR transmissions depends on whether guard-band reuse is possible or not. To illustrate, consider the channel status table in Fig. 4. Channels $\{2,6,15, \ldots, 19\}$ are idle. In the no guard-band reuse (guard-band reuse) case, only channels $\{16,17,18\}$ $(\{2,6,15,16,17,18\})$ are available for data transmissions by CR users. As explained in Section 3.2.2, this difference in guard band reservation makes channel assignment under both cases different. In this paper, we investigate the problem of channel/power allocation for both cases.

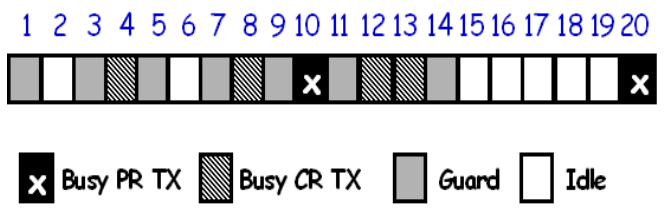

Fig. 4. Example that illustrates the impact of guard-band reuse. Remark 1: In general, the difference in the transmis- 
sion powers of two frequency-adjacent links impacts the required amount of guard-band separation. For classic multi-channel networks, such difference must be taken into account during the channel/power assignment process. However, for opportunistic CRNs, transmissions are already limited by stringent power masks, which ensure that a CR transmission does not cause unacceptable interference to adjacent-channel PR users. For example, the FCC imposes strict power limits on opportunistic communications over the TV white spaces (the spectral mask in an idle TV channel must be at least $55 \mathrm{~dB}$ below the highest average power used by PR users [18]). Accordingly, in our treatment, we assume that the power difference between two adjacent CR links is small.

\subsection{Problem Statement and Design Constraints}

We consider a CRN that uses an asynchronous multichannel CSMA/CA-like random access strategy, with a common control channel (CC) (e.g., [2], [3], [5], [19]). CSMA/CA-type protocols ensure that only one link (a transmitter-receiver pair) can access the $\mathrm{CC}$ at any given time. Other contending links have to wait until the $\mathrm{CC}$ becomes idle. Upon accessing the CC, the two communicating devices exchange control information, conduct channel assignment for their own transmission, and announce this channel assignment to their neighbors. Our channel assignment algorithm aims at minimizing the number of required channels for a given CR transmission, assuming that this transmission has already been requested over the CC. Specifically, for a given CR transmission, both the transmitter and receiver need to cooperatively select appropriate data channels and transmission powers over these channels while meeting the following constraints:

1) Half-duplex operation: While transmitting, a $C R$ user cannot receive/listen.

2) Fixed rate per channel: Each channel $i$ can support a transmission rate $W$ (in bps) if its received SINR is $\geq \mu^{*}$.

3) Exclusive channel occupancy: A selected data channel cannot be assigned to more than one transmission in the same neighborhood.

4) Rate demand: A CR transmission $j$ has a rate demand $R_{D}(j)=m_{j} W$, where $m_{j}$ is the number of required data channels $\left(m_{j} \leq M\right)$.

5) Maximum transmission power: For a given $C R$ transmission, the total transmission power $\left(P_{t o t}\right)$ over the selected channels is limited to $P_{\max }$.

6) Per-channel power constraint: The transmission power over an idle channel $i$ is limited to $P_{\max }^{(i)}$.

7) Guard-band reservation: A guard channel cannot be used for CR transmissions.

8) PR protection: To protect PR receptions, an idle channel that is adjacent to a channel occupied by a PR user cannot be used for CR transmissions [18]. In Fig. 4, channels $\{9,11,19\}$ cannot be used for data transmissions. Note, however, these channels can be used as guard bands for CR transmissions.
It is worth noting that performing channel and power assignment for multiple links at the same time (batch approach) provides better performance than performing the assignment sequentially (i.e., one link at a time). For a single collision domain (all nodes are within range of each other), the batch approach is feasible. However, the batch approach is not practical in a multi-hop environment, as it incurs high control overhead and delay. Specifically, to execute the channel/power assignment algorithm in a distributed manner, each CR user in a given contention region must first exchange rate demand, channel availability, and SINR information with other CR users in that region. This is impractical in a multihop network, given the rapid changes in channel dynamics that may take place while the information exchange is underway. Therefore, determining this optimal assignment incurs high message overhead and delay. Furthermore, the batch approach, while potentially useful for offline network planning, is not suitable for online asynchronous and distributed operation. Specifically, in our setup we consider an ad hoc CRN in which users generate their opportunistic transmission requests independently and asynchronously. Batching such requests requires imposing transmission synchrony to enable concurrent channel/power assignment (and consequently, concurrent data transmissions) over multiple links. This means deferring transmissions until a batch is formed, with negative implications on the delay performance of the underlying traffic. Even without considering the guard-band constraint, the joint power control/channel assignment problem for multiple links that aims at maximizing the overall network throughput is known to be NP-hard [20], [21].

\subsection{Problem Formulation}

The key idea behind our scheme is to minimize the number of required guard channels for a given transmission while relying only on information provided by the two communicating users. If multiple solutions exist, we seek the one that requires the least amount of transmit power.

Let $\mathcal{I}_{j}(t), \mathcal{G}_{j}(t)$, and $\mathcal{B}_{j}(t)$ denote, respectively, the sets of idle, guard, and busy channels, as seen by the $j$ th transmitter-receiver pair at any given time $t$. Note that these sets vary with time, and depend on the traffic dynamics of PR users. Because we focus on computing a feasible channel assignment $\Omega_{j} \subseteq \mathcal{I}_{j}(t)$ for a given transmission $j$ at time $t$, the subscripts $j$ and $t$ are dropped in the rest of this paper to simplify the notation. Given the current status of all channels (i.e., the sets $\mathcal{I}$, $\mathcal{G}$, and $\mathcal{B}$ ), the channel gain and interference over every channel $i \in \mathcal{I}$ as measured at the receiver of link $j$, the rate demand ( $m$ channels), and the SINR threshold $\mu^{*}$, the receiver of the $j$ th CR link can compute the minimum required power $\left(P_{i}\right)$ for every idle channel $i \in \mathcal{I}$ such that the received SINR is $\geq \mu^{*}$. Formally, the channel 
assignment problem can be stated as follows:

$$
\begin{gathered}
\underset{\Omega \subseteq \mathcal{I}}{\operatorname{minimize}}\left[k(\Omega)+\frac{P_{t o t}(\Omega)}{P_{\max }}\right] \\
\text { s.t. } \quad P_{t o t}(\Omega) \stackrel{\stackrel{\text { def }}{=}}{=} \sum_{i \in \Omega} P_{i} \leq P_{\max } \\
|\Omega|=m
\end{gathered}
$$

where $k$ is the number of frequency blocks assigned to the $j$ th link.

Note that the first constraint in (2) ensures that $\frac{P_{t o t}(\Omega)}{P_{\max }} \leq 1$ for any feasible assignment $\Omega$. So, for any two feasible assignments $\Omega_{1}$ and $\Omega_{2}$ with $k\left(\Omega_{1}\right)<k\left(\Omega_{2}\right)$, the above formulation will select $\Omega_{1}$ over $\Omega_{2}$, irrespective of $P_{t o t}$. If $k\left(\Omega_{1}\right)=k\left(\Omega_{2}\right)$, then the assignment that results in the minimum $P_{t o t}$ is selected. In other words, among all solutions that provide the minimum number of blocks and meet the rate demand and power constraints, we give preference to the one that minimizes the total transmission power.

For $i=0, \ldots, M+1$, define $\alpha_{i}$ as follows:

$$
\alpha_{i}= \begin{cases}1, & \text { if channel } i \in \Omega \\ 0, & \text { otherwise. }\end{cases}
$$

We let $\alpha_{0}=\alpha_{M+1}=0$. The number of frequency blocks for a given assignment $\Omega$ can now be written as:

$$
k(\Omega)=\frac{1}{2} \sum_{i=1}^{M+1}\left(\alpha_{i}-\alpha_{i-1}\right)^{2} .
$$

For all channels $i$ in $\mathcal{G} \cup \mathcal{B}$, we set $\alpha_{i}$ to 0 . Then, for each idle channel in $\mathcal{I}$, we compute the minimum required power $\left(P_{i}\right)$ needed to achieve the SINR threshold. If $P_{i}>$ $P_{\max }^{(i)}$, we set $\alpha_{i}$ to 0 . Let $\mathcal{I}^{*} \subseteq \mathcal{I}$ be the set of channels for which the required power $P_{i}$ is below the maximum power $P_{\max }^{(i)}$. Based on the above and substituting (4) into (2), the optimization problem becomes:

$$
\begin{gathered}
\underset{\left\{\alpha_{i}, i \in \mathcal{I}^{*}\right\}}{\operatorname{minimize}}\left[\frac{1}{2} \sum_{i=1}^{M+1}\left(\alpha_{i}-\alpha_{i-1}\right)^{2}+\sum_{i=1}^{M} \frac{P_{i}}{P_{\max }} \alpha_{i}\right] \\
\text { s.t. } \quad \sum_{i=1}^{M} \alpha_{i}=m \\
\sum_{i=1}^{M} \alpha_{i} P_{i} \leq P_{\max } .
\end{gathered}
$$

The optimization problem in (5) is a binary quadratic program (BQP).

Proposition 1: The optimization problem in (5) can be transformed into a binary linear programming (BLP) with a linear objective and linear constraints.

Proof: The BQP formulation in (5) can be easily transformed into BLP by introducing a new auxiliary variable $z_{i}, i=1, \ldots, M+1$ :

$z_{i} \stackrel{\text { def }}{=} \begin{cases}0, & \text { if channels } i \text { and } i-1 \text { have the same status } \\ 1, & \text { otherwise }\end{cases}$

and adding the following constraints on $z_{i}$ :

$$
\left\{\begin{array}{l}
z_{i} \geq \alpha_{i}-\alpha_{i-1} \\
z_{i} \geq \alpha_{i-1}-\alpha_{i}
\end{array}\right.
$$

According to (6), if channels $i$ and $i-1$ have the same status, then $z_{i}=0$. Otherwise, $z_{i}$ must be at the same time greater than or equal -1 and 1 . Thus, it must be 1 .

With the introduction of $z_{i}$, the quadratic term in the objective function in (5) can be changed to $\frac{1}{2} \sum_{i=1}^{M+1} z_{i}$. This results in the following (equivalent) formulation to the original BQP problem in (5):

$$
\begin{gathered}
\underset{\left\{\alpha_{i}, z_{i}\right\}, i \in \mathcal{I}^{*}}{\operatorname{minimize}}\left[\frac{1}{2} \sum_{i=1}^{M+1} z_{i}+\sum_{i=1}^{M} \frac{P_{i}}{P_{\max }} \alpha_{i}\right] \\
\text { s.t. } \quad \sum_{i=1}^{M} \alpha_{i}=m \\
\quad \sum_{i=1}^{M} \alpha_{i} P_{i} \leq P_{\max } \\
\alpha_{i}-\alpha_{i-1}-z_{i} \leq 0, i \in\{1, \ldots, M+1\} \\
-\alpha_{i}+\alpha_{i-1}-z_{i} \leq 0, i \in\{1, \ldots, M+1\} \\
\alpha_{i} \in\{0,1\}, i \in\{1, \ldots, M\} \\
z_{i} \in\{0,1\}, i \in\{1, \ldots, M+1\} .
\end{gathered}
$$

It is clear that the optimization problem in (7) is a BLP.

\section{Channel Assignment Schemes}

To assess the impact of guard-band awareness, we first present a greedy guard-band-unaware assignment scheme, whose simplicity and low processing overhead made it attractive for use in multi-channel systems [6], [22], [23]. However, this scheme results in a high blocking probability for data transmissions, leading to a reduction in network throughput. Subsequently, we propose a novel guard-band aware spectrum sharing algorithm that improves CRN throughput performance.

The greedy guard-band unaware algorithm aims at selecting the "best" $m$ channels that combinedly guarantee a normalized rate demand $m$. Recall that in our setup every selected channel delivers the same basic rate, but using a transmission power that differs from one channel to another. Because the greedy algorithm is indifferent to guard-bands, the only possible way to optimize its selection is by minimizing the total transmission power subject to the constraint $P_{\max }$. On the other hand, our guardband-aware spectrum sharing algorithm (described in Section 3.2) also selects $m$ channels (each supporting the same basic rate), but while being aware of the guard-band overhead and, secondarily, the total used power. So both algorithms achieves the same rate demand $m$ and satisfy the power constraint $P_{\max }$. They differ in the objective function, which in the case of our algorithm is cognizant of the guard-band overhead.

\subsection{Greedy Algorithm}

The greedy approach proceeds in three steps:

1) Consider a given prospective $C R$ link. Given $\mathcal{I}, \mathcal{G}, \mathcal{B}$, the channel gains, the measured interference over every channel $i \in \mathcal{I}$ along the given $\mathrm{CR}$ link, and $\mu^{*}$, 
calculate the required power $P_{i}, \forall i \in \mathcal{I}$. If $P_{i} \leq P_{\max }^{(i)}$, then channel $i$ is placed in the set $\mathcal{I}^{*}$.

2) Sort the channels in $\mathcal{I}^{*}$ increasingly according to $P_{i}$.

3) Select the first $m$ channels from the top of the sorted list. If the total transmission power over the best $m$ channels exceeds $P_{\max }$, then there is no feasible channel assignment.

Lemma 1: For a given $C R$ transmission with a given rate demand, if the greedy algorithm results in an infeasible solution, then there is no feasible solution to the problem. The proof is trivial and is omitted for brevity.

\subsection{Sub-optimal Algorithm Based on Sequential Fix- ing}

The BLP in (7) is a combinatorial problem. Its solution is, in general, NP-hard. There exist several methods for approximately solving BLP problems, including cutting plane methods, decomposition methods, and branchand-bound methods [24]. However, the worst-case time complexity of such approximations is still exponential. Instead, by exploiting the special structure of the problem, we develop a polynomial-time sub-optimal algorithm. Specifically, we relax the binary constraints $\alpha_{i} \in\{0,1\}$ and $z_{i} \in\{0,1\}$ into real numbers in $[0,1]$. The resulting linear relaxation (LR) is solvable in polynomial time [25]. The main idea behind our fast solution is to fix the values of $\alpha_{i}$ sequentially through solving a series of relaxed LP problems, with at least one $\alpha_{i}$ finalized to a binary value in each iteration. Our sub-optimal algorithm is called sequential fixing LP (SFLP). Two variants of the SFLP algorithm are presented. The first variant does not allow guard-band sharing, whereas the second one allows it. We here note that sequential fixing has been previously used in [26] to solve integer programming problems. The main difference between our algorithm and the one in [26] is that in [26] there is no guarantee that a feasible solution can be found. Our algorithm improves upon [26] by adding a revised fixing phase when any intermediate fixing leads to infeasibility, and hence a feasible solution can always be found.

\subsubsection{SFLP-based Assignment with No Guard-band Reuse}

In the first iteration of the assignment scheme, we relax the binary constraints by allowing $\alpha_{i}$ 's and $z_{i}$ 's to take real values in $[0,1]$. For any unavailable (guard or busy) channel, we set $\alpha_{i}=0$ (i.e., these channels cannot be assigned to a new CR transmission). We also set $\alpha_{i}=0$ for any idle channel that is adjacent to a busy channel occupied by a PR user or to an already allocated guard channel. We refer to the resulting formulation as $\mathrm{LR}^{(1)}$, which must have a feasible solution if the original BLP has a feasible solution (i.e., if $\mathrm{LR}^{(1)}$ problem is infeasible, then there is no feasible channel assignment). The solution to $\mathrm{LR}^{(1)}$ provides a lower bound on the optimal solution to (7), because the feasibility region of the BLP is a subset of that of $\mathrm{LR}^{(1)}$. However, the solution of $\mathrm{LR}^{(1)}$ is, in general, not a feasible solution to the original BLP problem, because $\alpha_{i}$ 's and $z_{i}{ }^{\prime}$ s can now take real values between 0 and 1 . Among all newly obtained real-valued $\alpha_{i}{ }^{\prime}$ s, we then set the one that has the largest value to 1 . For the $j$ th iteration $j=2, \ldots m$, the algorithm proceeds as follows:

i. Relax all unfixed $\alpha_{i}$ 's and all $z_{i}$ 's to real values in $[0,1]$.

ii. Check the feasibility region of the new LR, called $\mathrm{LR}^{(j)}$. If this region is empty, this means the fixing in the $(j-1)$ th iteration was not correct. In this case, flip the value of the last fixed variable to 0 and update $\operatorname{LR}^{(j)}$. Note that the revised $\mathrm{LR}^{(j)}$ problem must be feasible (see Lemma 3).

iii. Solve the modified $\operatorname{LR}^{(j)}$, whose variables do not include those that have been fixed after the execution of $\mathrm{LR}^{(j-1)}$.

iv. Choose the largest $\alpha_{i}$ and fix it to 1 .

v. $\quad$ Repeat the above process until a total of $m \alpha_{i}{ }^{\prime}$ s are set to 1 (feasible assignment) or all $\alpha_{i}{ }^{\prime}$ s are fixed and no feasible channel assignment can be found. Pseudo-code of the SFLP algorithm is given in Algorithm 1.

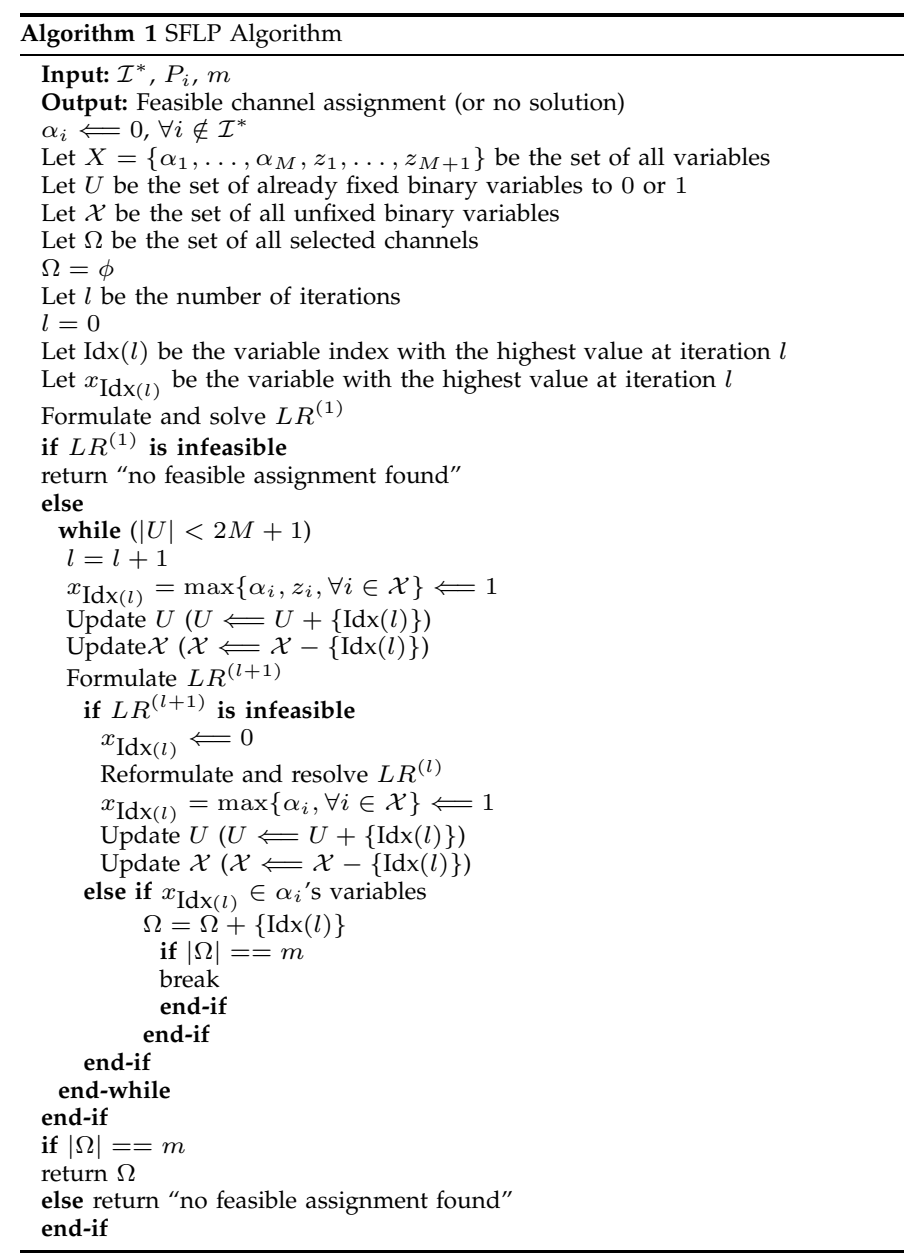

\subsubsection{SFLP-based Assignment with Guard-band Reuse}

Now, we consider the case with guard-band reuse. When guard-band sharing is not allowed, minimizing the number of frequency blocks is equivalent to minimizing the 
number of newly introduced guard channels. However, when guard-band reuse is allowed, the number of introduced guard channels is minimized by attempting to reuse existing guard channels and at the same time minimize the number of frequency blocks required for a given transmission. To achieve $100 \%$ efficiency, we should select frequency blocks that do not introduce any additional guard channels (i.e., already has a guard channel on each side and can reuse it). To illustrate, consider the channel status table in Fig. 4. Suppose that a prospective $\mathrm{CR}$ transmission requires 2 data channels. Assume that any possible combination of two idle channels is power-feasible (i.e., $P_{t o t} \leq P_{\max }$ ). Suppose that channels 16 and 17 require the minimum $P_{t o t}$ among all possible combinations of two-adjacent channels. According to the SFLP algorithm in Section 3.2.1, channels 16 and 17 will be selected. This assignment introduces 2 additional guard channels ( $50 \%$ spectrum efficiency). However, when guard-band reuse is allowed, by selecting channels 2 and 6 , no additional guard channels will be introduced, leading to $100 \%$ spectrum efficiency.

To incorporate guard-band reuse, we modify the SFLP algorithm, as follows. In the first iteration, we relax the binary constraints by allowing $\alpha_{i}$ 's and $z_{i}$ 's to take real values in $[0,1]$. For a busy channel $i \in \mathcal{B}$ (occupied by a PR or CR user), we set $\alpha_{i}=0$. We also set $\alpha_{i}=0$ for all idle channels that are adjacent to a busy channel occupied by a PR user. For every guard channel $i \in \mathcal{G}$, we set $\alpha_{i}=1$. By setting $\alpha_{i}=1 \forall i \in \mathcal{G}$, our algorithm gives preference to frequency blocks that already have guard channels reserved by neighboring CR transmissions. Because $\alpha_{i}$ is set to $1 \forall i \in \mathcal{G}$, the constraint on the number of selected channels in the original BLP should be updated as follow: $\sum_{i=1}^{M} \alpha_{i}=m+|\mathcal{G}|$. We refer to the resulting formulation as $\mathrm{LR}^{(1)}$, which must have a feasible solution if the modified BLP has a feasible solution. Among all $\alpha_{i}$ 's of the optimal solution of $\mathrm{LR}^{(1)}$, we set the one that has the largest value to 1 . Then, for the subsequent iterations $(j=2, \ldots m)$, the same algorithm used for SFLP with no guard-band reuse is used to compute a feasible channel/power assignment. In the rest of this paper, we refer to the channel assignment mechanism that uses the modified SFLP algorithm as SFLP-GR, and the one that uses the original SFLP algorithm as SFLP.

Lemma 2: If the greedy solution in Section 3.1 is feasible, then the original BLP and the corresponding LR (i.e., $\mathrm{LR}^{(1)}$ ) have feasible solutions.

Proof: The feasibility regions of the BLP and $\mathrm{LR}^{(1)}$ include the greedy solution (i.e., the greedy solution is one of the possible combinations of the binary variables that need to be examined to solve the BLP problem).

Lemma 3: The updated $\mathrm{LR}^{(j)}$ problem in Step (ii) of our algorithm must be feasible.

Proof: (By induction). In the second iteration, if $\mathrm{LR}^{(2)}$ has no feasible solution, it will be updated by switching the value of the last fixed variable to 0 . The total transmission power will therefore decrease. Consequently, the total transmission power constraint will not be violated. Thus, the updated $\mathrm{LR}^{(2)}$ must have at least one feasible solution. In the second iteration, $\mathrm{LR}^{(2)}$ comes from either a feasible $\mathrm{LR}^{(2)}$ or an updated feasible $\mathrm{LR}^{(2)}$ of the first iteration. Thus, $\mathrm{LR}^{(2)}$ must be feasible in the second iteration. Given that $\mathrm{LR}^{(2)}$ is feasible in the second iteration, the rationale used in proving the feasibility of the first iteration also applies here to prove the feasibility of $\mathrm{LR}^{(3)}$ in the third iteration. This induction is repeated for all iterations. Noting that all variables are bounded in $[0,1]$, Lemma 3 holds.

Theorem 1: The SFLP algorithm returns a feasible solution or determines that no feasible solution exists in no more than $\max \left\{m,\left|\mathcal{I}^{*}\right|\right\}$ iterations.

Proof: The proof follows from Lemma 2 and 3. It is guaranteed that in each iteration, one new $\alpha_{i}$ variable will be fixed to either 0 or 1 and a new feasible LR will be generated for the next iteration. If all the generated $\mathrm{LR}^{(j)}$ problems are feasible, then $m$ iterations are required. Otherwise, a maximum of $\left|\mathcal{I}^{*}\right|$ iterations are required to determine whether $m$ feasible channels can be found.

Based on Theorem 1, it is easy to show that the time complexity of the SFLP algorithm is bounded by the complexity of the LR solver times $\max \left\{m,\left|\mathcal{I}^{*}\right|\right\}$. Because an LR solver (LP solver) has a polynomial complexity, the complexity of our sequential fixing algorithm is also polynomial. Our simulations show that in most cases our algorithm requires $m$ iterations to find a feasible assignment. In addition, the performance gap between the SFLP solution and the optimal solution (obtained through an exhausted search) is shown to be very small (below 5\%), and in most cases it is zero. A lower bound on the optimal BLP solution is provided by the solution to $\mathrm{LR}^{(1)}$ in the first iteration. Our simulations show that this bound is typically loose.

\section{Channel Access Protocol}

\subsection{Protocol Overview}

We integrate our guardband-aware SFLP channel assignment algorithm into a distributed multi-channel MAC protocol for CRNs with a common control channel. The main purpose of this protocol is to realize the proposed channel assignment algorithm in a distributed manner (i.e., resolve channel contention between contending CR pairs, exchange control information needed to perform channel assignment, and announce the outcome of this channel assignment to neighboring devices). Our MAC protocol differs from previous designs in that it exploits the "multi-receive multi-transmit" capability of radios (i.e., each radio is capable of receiving/transmitting over multiple channels simultaneously). The operation, however, is half-duplex, i.e., while transmitting, the radio cannot receive/listen, even over other channels. In particular, a user that is not transmitting any data can tune its radio to the control channel. This way, the multi-channel hidden-terminal problem can be alleviated.

\subsection{Operational Details}

To facilitate multi-channel contention resolution and reduce the likelihood of collisions, each CR user, say $A$, 
maintains an available-channel list (ACL), a guardbandchannel list (GCL), and a busy-node list (BNL). ACL( $A$ ) represents the set of all idle channels $\mathcal{I}$ that are not occupied (or reserved as guard channels) by other CR users within $A^{\prime}$ 's one-hop communication range. $\mathrm{GCL}(A)$ represents the set of channels $\mathcal{G}$ that have been reserved as guard bands within $A^{\prime}$ s one-hop communication range. $\operatorname{BNL}(A)$ consists of the IDs of users that are currently busy transmitting/receiving data packets in $A^{\prime}$ 's neighborhood. The $\operatorname{ACL}(A), \operatorname{GCL}(A)$, and $\operatorname{BNL}(A)$ lists are continuously updated according to the channel access dynamics and overheard control packets (the distributed acquisition of the ACL, GCL, and BNL information is discussed in Section 4.4). The MAC protocol uses similar interframe spacings and collision avoidance strategies of the 802.11 scheme (implemented here over the control channel), including physical carrier sensing and random backoff before initiating control-packet exchanges. Upon accessing the control channel, communicating users perform a three-way handshake, during which they exchange control information, conduct the channel assignment, and announce the outcome of this channel assignment to their neighbors.

\subsection{Channel Access Mechanism}

Consider a node $A$ that has a data packet of size $D_{p k t}(A)$ to transmit to user $B$. The rate demand $R_{d}(A)=m W$. For its first transmission or following a successful data transmission, node $A$ selects a random backoff duration $B_{O F F}(A)$ that is uniformly distributed between $B_{\min }$ and $B_{\max }$, where $B_{\min }$ is the minimum backoff duration (has to be larger than the SIFS duration) and $B_{\max }$ is the maximum backoff duration. $B_{\max }$ is measured in units of control-packet transmission times, and is generally much smaller than the minimum contention window $\left(C_{\text {Wmin }}\right)$, typically used in the classic (single-channel) 802.11 protocol.

While the backoff timer is frozen (control channel is busy), CR user $A$ continues to listen to, and possibly receive over, the control channel. If $A$ overhears a clearto-send (CTS) or decide-to-send (DTS) packet (explained below) from any other node, it updates $\operatorname{ACL}(A), \operatorname{GCL}(A)$, and $\operatorname{BNL}(A)$. If $\operatorname{ACL}(A)$ is still nonempty and the intended receiver is not in $\operatorname{BNL}(A), A$ continues to decrease its timer; otherwise, it freezes its timer until new data channels become available or until the intended receiver finishes its data transmission/reception. If $A$ overhears an RTS (CTS) packet, it freezes its timer for the duration of that control exchange plus the SIFS duration. This ensures that $A$ will not attempt to transmit a control packet in the period between the current overheard RTS (CTS) and the subsequent CTS (DTS) (which node $A$ may not be able to hear). If $A$ captures the control channel (i.e., does not sense a carrier over the control channel for the randomly selected backoff period $B_{O F F}(A)$ ), it proceeds as follows:

- $A$ sends an RTS message at power $P_{\max }$. The RTS packet includes $\operatorname{ACL}(A), \operatorname{GCL}(A)$, and $R_{d}(A)$ (or equivalently $m$ ).
- $A^{\prime}$ 's neighbors other than $B$ who can correctly decode the RTS will stay silent until either they receive a DTS packet from $A$ or until the expected time for the DTS packet has passed.

- Upon receiving the RTS packet, $B$ proceeds with the channel assignment process.

- Depending on the outcome of the channel assignment process, $B$ decides whether or not $A$ can transmit. If not, then $B$ does not respond to $A$, prompting $A$ to back off following the same backoff procedure described before (i.e., selecting a new value for $B_{O F F}(A)$ between $B_{\min }$ and $\left.B_{\max }\right)$, and retransmit the RTS later. A limit of RTSmax is imposed on the number of RTS retransmissions. If this limit is reached, the transmitter concludes that the intended receiver is down. It reports an error to the higher layer and drops the MAC frame from its queue. If there is a feasible channel assignment, $B$ sends a CTS message to $A$ that contains the assigned channels, denoted by $\Omega(A, B)$, the transmit powers over the selected channels, and the duration $\left(T_{p k t}(A)\right)$ needed to reserve the assigned channel. The CTS implicitly instructs $B^{\prime}$ s neighbors to refrain from transmitting over the assigned channel for the duration $T_{p k t}(A)$.

- Once $A$ receives the CTS, it replies back with a DTS message, informing its neighbors of $\Omega(A, B)$ and $T_{p k t}(A)$. Such a three-way handshake is typically needed in multi-hop multi-channel CSMA/CA protocols (e.g., [6], [22], [23]). For single-collision domain networks, where all users can hear each other, there is no need for the DTS packet. Likewise, in singlechannel multi-hop networks, the DTS packet is also not needed.

- After completing the RTS-CTS-DTS exchange, the transmission $A \rightarrow B$ proceeds. Once successfully completed, $B$ sends back an ACK packet to $A$ over the $m$ assigned data channels.

Because there is no interference between data and control packets, a user who hears the RTS (CTS) packet defers transmitting only until the end of the control handshaking process. This allows for more parallel transmissions to take place in the same vicinity.

\subsection{Protocol Overhead}

A key feature of the MAC design in previous section is that it is based on passive learning. Specifically, when not transmitting/receiving, CR users keep listening to the control channel and overhearing control-packet exchanges, including those intended for other nodes (i.e., monitoring the dynamics of other CR users). From these exchanges, users obtain channel-usage information and update their ACL, GCL, and BNL tables in a distributed manner. Thus, our MAC protocol does not require transmitting additional control messages beyond those used in the three-way handshake, which is needed anyway in any multi-channel CSMA/CA MAC protocol [2], [22], [23]. In fact, except for the GCL list (which is included only in the RTS packet), all the control information included inside 
the RTS/CTS/DTS packets is part of any multi-channel CSMA/CA MAC protocol. Note that the BNL list is not conveyed inside the RTS/CTS/DTS packets. The format of the RTS, CTS, and DTS packets are as follows:

$$
\begin{aligned}
& \operatorname{RTS}(A \rightarrow B)=\left\{A, B, \operatorname{ACL}(A), \operatorname{GCL}(A), R_{d}(A), D_{p k t}(A)\right\} . \\
& \operatorname{CTS}(B \rightarrow A)=\left\{B, A, \Omega(A, B), P_{i}, \forall i \in \Omega(A, B), T_{p k t}(A)\right\} . \\
& \operatorname{DTS}(A \rightarrow B)=\left\{A, B, \Omega(A, B), T_{p k t}(A)\right\} .
\end{aligned}
$$

\subsection{Protocol Limitations and Solutions}

\subsubsection{Transmitter Deafness}

While receiving a data packet, a user continues to listen to control exchanges taking place over the control channel, and hence can update its ACL, GCL, and BNL accordingly. However, a user that is transmitting a data packet will not be able to listen to the control channel, so its ACL, GCL, and BNL may become outdated. This transmitter deafness problem is primarily caused by the half-duplex nature of the radios. To remedy it, when the receiver sends its ACK, it includes in this ACK any changes in the ACL, GCL, and BNL that may have occurred during the transmission of the data packet. The transmitter uses this information to update its own lists.

\subsubsection{Incomplete Channel Availability Information}

Just before completing the receipt of a data packet, a node may start receiving a control packet over the control channel. To avoid interrupting the reception of the control packet, the receiving node defers the transmission of its ACK packet, i.e., a time gap is inserted between the end of the data packet and the start of its associated ACK. Note that sending the ACK packet directly after the data packet may result in inaccurate ACL and GCL at both the receiver and transmitter, and may lead to subsequent data collisions. The time gap depends on the type of the control packet. If the control packet is a CTS or DTS, the node will start its ACK transmission on the data channels right after completely receiving that control packet. If the control packet is an RTS, the receiver will wait until the end of the next control packet (potentially, a CTS or DTS) and then send the ACK. This design significantly reduces collisions due to incomplete channel availability information. Accordingly, following the transmission of the data packet, the transmitting node sets its ACK timer to the duration of two control packets plus an ACK duration.

\subsubsection{Inconsistency in the ACL/GCL Tables}

Even if the RTS-CTS-DTS exchange was conducted successfully and data packet transmission ensued over some chosen data channels, a collision over the data channel is still possible due to inconsistencies between the ACL/GCL tables of different nodes and also due to unaccounted for interference from outside the CRN (i.e., due to the dynamics of PR activities). If that happens, the receiver will not send the ACK packet, triggering a retransmission of the data packet. We set a limit DATAmax on the number of data-packet retransmissions. For each data-packet retransmission, the complete contention process has to be repeated. It should be noted that the conventional exponential backoff approach (i.e., doubling the value of $B_{\max }$ after each collision) is not adopted in our design, as such an approach is deemed conservative for a multi-channel environment with a dedicated control channel.

\subsubsection{Effect of Losing Control Packets}

The effect of losing an RTS or CTS packet on network performance is the same for our protocol as for any multichannel protocol. The loss of either packet is detected at the transmitter through a timeout mechanism. Because a backoff mechanism is used, each loss leads to increased delay in the transmission process. For neighboring $C R$ users, the loss of an RTS/CTS/DTS packet can result in inconsistencies between the ACL tables of different nodes, which leads to collisions over data channels and consequently increases the number of data retransmissions. For each data retransmission, the complete contention process has to be repeated, which leads to increased delay in the transmission process.

\subsubsection{Control Information Exchange}

Establishing control communications in an opportunistic DSA network (the so-called "rendezvous" problem) is a very important problem, which has been addressed in several works (e.g., [27]-[29]). In principle, establishing initial control communications can be done through a designated channel or by using frequency hopping (FH). Despite its significance, we believe the rendezvous problem is outside the scope of our paper. So for simplicity, in our protocol design we assumed that control communications take place over a common control channel. Integrating an FH-based rendezvous scheme into our protocol is rather straightforward. Note that pre-specifying a common control channel for the initial dialogue does not necessarily mean dedicating this channel to the CRN. For example, the initial control dialogue can be conducted over an unlicensed ISM band, enabling two CR nodes to exchange channel availability information. Subsequently, the two nodes can decide on how to use their common spectrum holes. In fact, this has been the philosophy behind many previous MAC designs for CRNs (e.g., [6], [7], [30]).

\section{Performance Evaluation}

\subsection{Simulation Setup}

We consider $N$ CR links in a 100 meter $\times 100$ meter area. We assume that there are $M=21$ channels, each licensed to one PRN. CR users can opportunistically access the 21 channels. Each channel has $1 \mathrm{MHz}$ of bandwidth. The carrier frequency of the $i$ th PRN is $f_{i}=900+i \mathrm{MHz}$, for $i=1, \ldots M$. We set $\mu^{*}$ to 0.63 for all channels. The status of a PR signal is modeled as a 2-state Markov model that alternates between IDLE and BUSY states. A BUSY (IDLE) state indicates that some (no) PR user is transmitting over the given channel. For channel $i$, 

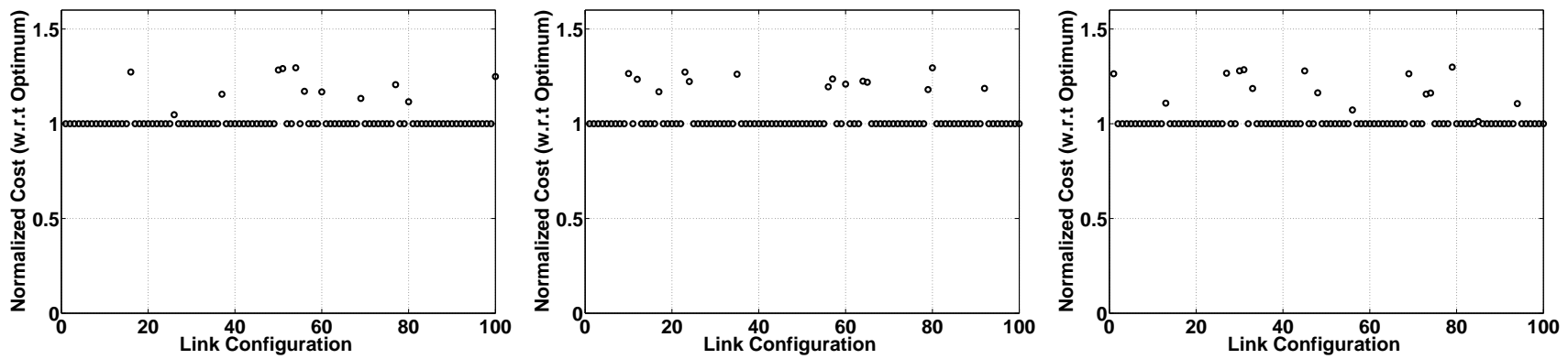

(a) Low activity $\left(P_{B}=0.1\right.$, average $=1.0287$, (b) Moderate activity $\left(P_{B}=0.4\right.$, average $=(\mathrm{c})$ High activity $\left(P_{B}=0.7\right.$, average $=$ variance $=0.0059$ ) 1.0425 , variance $=0.0074$ ) 1.0291 , variance $=0.0052$ )

Fig. 5. Normalized cost of the SFLP solution relative to the optimal solution $(m=4)$.

we denote the average IDLE and BUSY durations by $\lambda_{i}$ and $\mu_{i}$, respectively. In any given slot, the $i$ th PRN is active with probability $P_{B}^{(i)}=\frac{\mu_{i}}{\lambda_{i}+\mu_{i}}$. We set $\mu_{i}=100 \mathrm{~ms}$ and $\lambda_{i}=\lambda \forall i \in\{1, \ldots, M\}$. Accordingly, $P_{B}^{(i)}=P_{B}, \forall i$. We consider a Rayleigh fading model for the channel between any two users. Specifically, for a transmitterreceiver separation $d$, the received power over the $i$ th channel is given by:

$$
P_{r}^{(i)}=P_{o}^{(i)}\left(\frac{d}{d_{o}{ }^{(i)}}\right)^{-4} \xi^{(i)}, \quad d \geq d_{o}{ }^{(i)}
$$

where $P_{o}^{(i)}=\frac{P_{t}^{(i)} G_{t}^{(i)} G_{r}^{(i)} l_{i}^{2}}{\left(4 \pi d_{o}^{(i)}\right)^{2}}$ is the path loss of the closein distance $d_{o}^{(i)}=\max \left\{\frac{2 D^{2}}{l_{i}}, D, l_{i}\right\}, D$ is the antenna length, $P_{t}^{(i)}$ is the transmission power, $G_{t}^{(i)}$ is the antenna gain at the transmitter, $G_{r}^{(i)}$ is the antenna gain at the receiver, $l_{i}$ is the wavelength of $f_{i}$, and $\xi^{(i)}$ is a normalized random variable that represents the power gain of the fading process. For Rayleigh fading, $\xi^{(i)}$ is exponentially distributed. We set the maximum transmission power of a CR user to $P_{\max }=1 \mathrm{~W}$, the thermal noise power density to $10^{-21} \mathrm{~W} / \mathrm{Hz}$ for all channels, and the antenna length to $D=5 \mathrm{~cm}$.

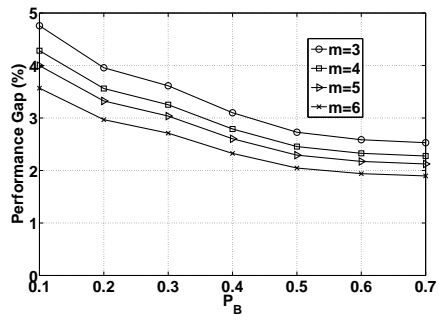

(a) Relative performance gap

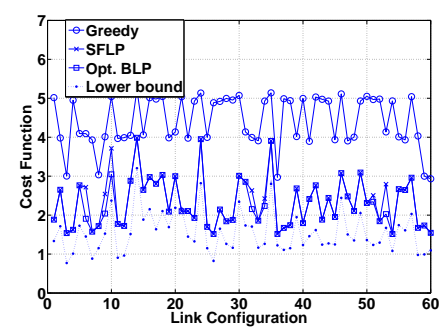

(b) Cost function $(m=4)$
Fig. 6. Performance gap between the SFLP solution and the optimal solution.

\subsection{Results}

\subsubsection{Link-level Simulations}

First, we use MATLAB simulations to empirically verify the validity of our SFLP algorithm and highlight its

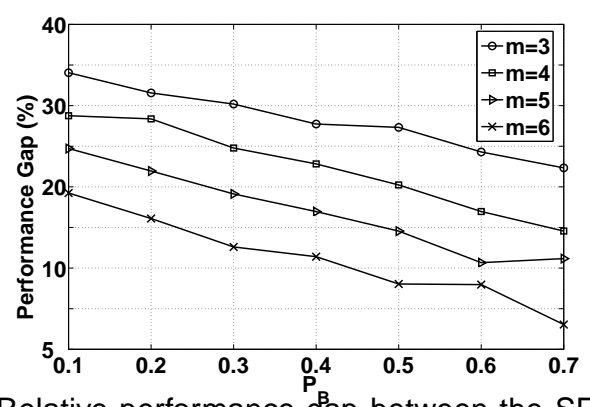

Fig. 7. Relative performance gap between the SFLP solution and the lower bound.

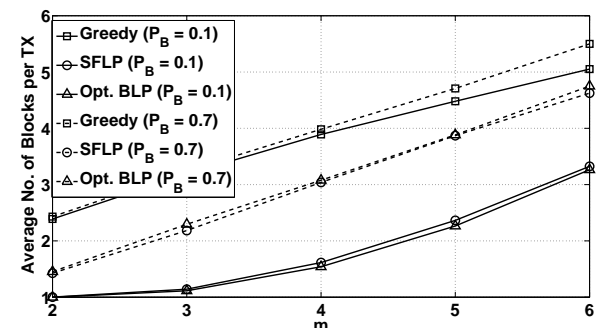

Fig. 8. Average number of assigned blocks vs. $m$.

advantages. We consider a single CR link, and investigate the performance of the SFLP algorithm as a function of various system parameters. The simulation results are presented for 100 "link configurations" (i.e., optimization instances). For each configuration, the source-destination distance is randomly generated. The SFLP algorithm is used to determine the channel assignment and the "cost function" (number of non-contiguous frequency blocks plus the normalized total transmission power). We compare these results with the lower bound (the solution for $\mathrm{LR}^{(1)}$ ), the optimal solution, and the greedy solution.

For $m=4$ and for different values of $P_{B}$, Fig. 5 depicts the normalized cost of the SFLP algorithm, relative to the optimal cost obtained through exhaustive search. In most cases, the SFLP solution is identical to the optimal solution. The mean normalized cost of the SFLP algorithm are $\leq 1.04$ and its variance is $\leq 0.007$. Hence, the SFLP algorithm achieves a near-optimal solution.

In Fig. 6(a), we plot the performance gap between the 


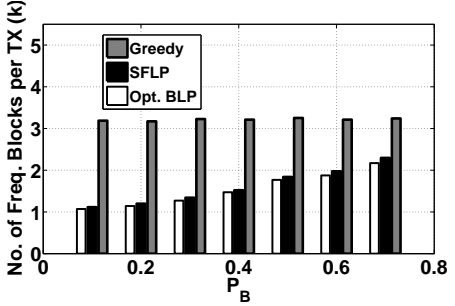

(a) $m=3$

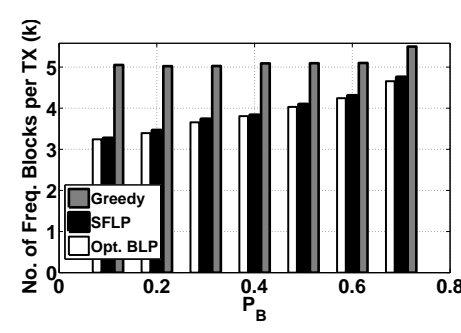

(b) $m=6$
Fig. 9. Average number of assigned blocks vs. $P_{B}$ for different values of $m$.

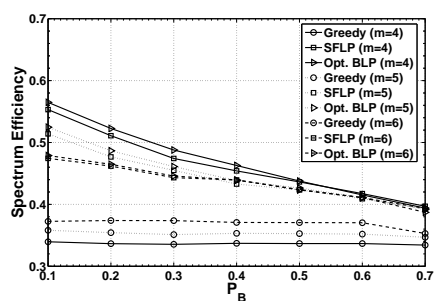

(a) Spectrum efficiency vs. $P_{B}$

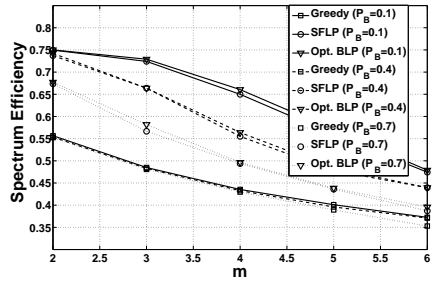

(b) Spectrum efficiency vs. $m$
Fig. 10. Spectrum efficiency under various channel assignment schemes.

sub-optimal SFLP solution and the optimal (but computationally expensive) solution. The gap never exceeds $5 \%$. In most cases, the two solutions are identical. The cost (objective function) is plotted in Figure 6(b) for 60 optimization instances. It is clear that the SFLP algorithm gives near-optimal performance (in line with the results in Fig. 5).

Because of its prohibitive computational complexity, the optimal solution can be obtained only for small networks. Thus, we also provide a lower bound on the performance (cost value) of the optimal solution. The cost value of the optimal solution lies between this lower bound and the cost value of the SFLP solution. This bound is obtained after the first iteration of the SFLP algorithm. Fig. 7 plots the performance gap between the SFLP solution and the lower bound. The gap becomes smaller at larger values of $m$ and $P_{B}$. This can be explained as follows. For a given set of idle channels, a larger $m$ (also, a larger $P_{B}$ ) decreases the number of possible channel combinations that can support the rate demand, and consequently increases the chances that the optimal and SFLP algorithms will select the same set of channels.

Fig. 8 illustrates the average number of non-contiguous frequency blocks $(k)$ as a function of the rate demand for two values of $P_{B}: 0.1$ and 0.7. The SFLP and the optimal BLP solutions have comparable performance in terms of $k$, and they significantly outperform the greedy approach. The greedy approach requires roughly the same $k$, irrespective of $P_{B}$. This is because it always selects the best available $m$ channels to support the rate demand. For both SFLP and optimal BLP, $k$ is smaller at smaller $P_{B}$ (see Fig. 9). This is because a smaller $P_{B}$ increases the number of idle channels, and consequently increases the chances of finding contiguous channels to support the rate demand. Fig. 9 indicates that for a given $P_{B}$, a larger value of $m$ results in a larger $k$.

Fig. 10 depicts the spectrum efficiency, expressed in terms of the ratio of the number of data channels to the number of assigned data-plus-guard channels, as a function of $P_{B}$ and $m$. The SFLP and the optimal BLP achieve comparable performance. They significantly outperform the greedy scheme. As demonstrated, spectrum efficiency decreases with $m$. This is expected since with a larger $m$, the likelihood of finding contiguous channels is lower. This increases the number of required guard channels, which consequently reduces spectrum efficiency.

\subsubsection{Network-level Simulations}

We conducted extensive simulations to investigate the performance of our proposed assignment approach when integrated into a distributed MAC protocol. We use the same simulation setup described in Section 5.1, but we vary $N$ (multi-user environment). Our network-level simulations are based on CSIM programs (a C-based, process-oriented, discrete-event simulation package [31]).

We compare our scheme with the optimal and the greedy schemes. For a fair comparison, in all of the tested assignment algorithms, we employ the same channel access protocol, described in Section 4. Each CR sender generates fixed-size $(2 \mathrm{~KB})$ data packets and requires $m$ data channels. The time is divided into slots, each corresponding to the transmission of one packet at a rate of $m \mathrm{Mbps}$. We assume that there is always a packet to transmit for each CR user. The locations of the CR transmitters and receivers are randomly assigned within the simulation region. In any given slot, the PR activity over a given channel is determined according to the 2-states Markov model described in Section 5.1. Our performance metrics include: (1) network throughput, (2) CR blocking rate, and (3) average energy consumption for successfully transmitting one data packet $\left(E_{p}\right)$. The CR blocking rate is defined as the percentage of transmission requests (packets in this case) that are blocked due to the unavailability of a feasible channel assignment. The results presented below are based on the average of 25 randomly generated topologies, with a simulation time of 10000 time slots for each topology.

\section{Channel Assignment with No Guard-band Reuse}

We first simulate a CRN where no guard-band reuse is allowed. We study the throughput performance as a function of $N, m$, and $P_{B}$. Figures 11 and 12 show that the SFLP algorithm significantly reduces the packet blocking rate and improves the overall throughput by up to $38 \%$ compared with the greedy approach. In all cases, the SFLP solution is within $5 \%$ of the optimal one. Fig. 12 reveals that the throughput gain of SFLP over the greedy approach is smaller at larger $P_{B}$. This is expected since the larger the value of $P_{B}$, the lower the chances of finding contiguous channels. This increases the number of required guard channels, and consequently reduces the 
throughput gain. Note that for large values of $m$ and $P_{B}$, all schemes achieve comparable throughput. In Fig. 13, we investigate the impact of various channel assignment strategies on $E_{p}{ }^{3}$. The greedy approach results in lower energy consumption because it always selects channels of high qualities. In other words, the throughput advantage of SFLP comes at the expense of additional energy consumption.

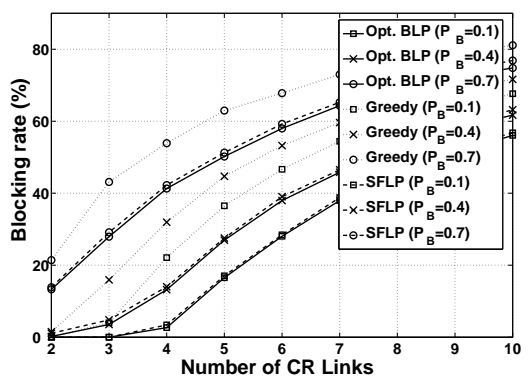

(a) $m=2$

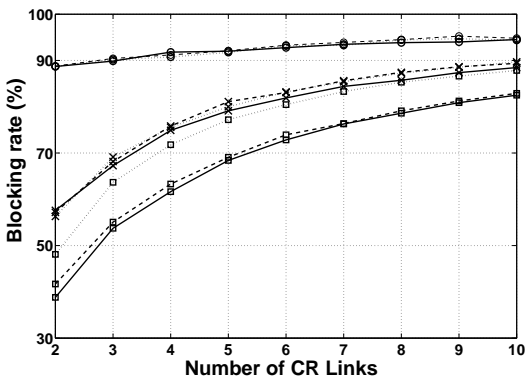

(b) $m=5$

Fig. 11. Blocking rate vs. $N$ for different values of $P_{B}$ (without guard-band reuse).

\section{Channel Assignment with Guard-band Reuse}

In this case, our proposed SFLP-GR scheme is compared with two other assignment schemes: the original SFLP (which tries to minimize the number of frequency blocks) and the greedy scheme. We adapt the operation of both schemes such that a guard channel can be reused (i.e., an idle channel that is adjacent to an already assigned guard channel can be used for CR data transmissions). We first study the throughput performance. Figures 1416 show that SFLP-GR significantly outperforms the other two schemes. SFLP-GR reduces the CR blocking rate and improves the overall throughput by up to $180 \%$ compared with the greedy approach, and $110 \%$ compared with the SFLP algorithm (with $m=4$ and $N=10$ ). This improvement is mostly attributed to its channel assignment, which attempts to reuse already allocated guard channels. Consequently, SFLP-GR preserves more channels for future CR transmissions, leading to an increase in the number of simultaneous transmissions. Fig.

3. The performance in terms of $E_{p}$ under SFLP is comparable with the one for the optimal solution. Thus, for clarity, Fig. 13 does not show the energy consumption of SFLP.

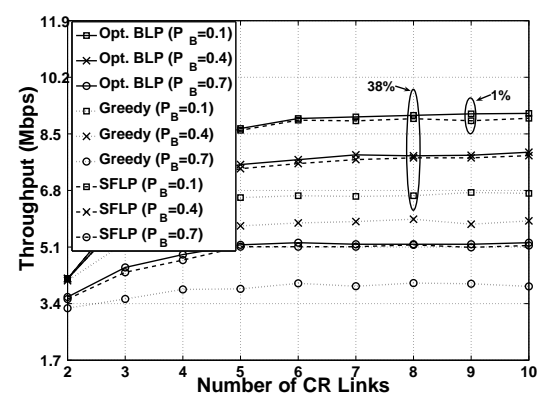

(a) $m=2$

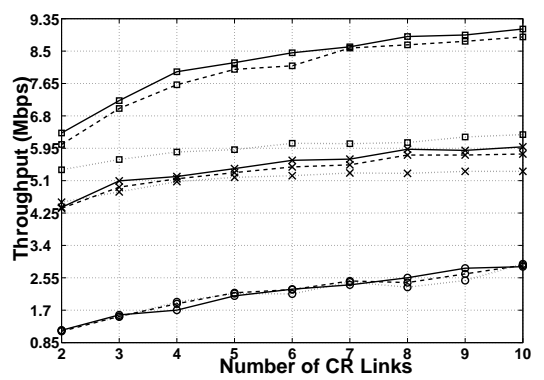

(b) $m=5$

Fig. 12. Throughput vs. $N$ for different values of $P_{B}$ and $m$.

16 shows that the achieved throughput is smaller at larger values of $m$. This is expected because a larger $m$ reduces the chances of finding $m$ contiguous idle channels to support the rate demand of a given CR transmission, and consequently increases the number of noncontiguous "frequency blocks" assigned to this transmission. This increases the number of required guard bands, and thus reduces the number of idle channels available for potential future CR transmissions resulting in a reduction in throughput gain. Similar to the case of no guard-band reuse, Fig. 16 also shows that the achieved throughput is smaller at larger values of $P_{B}$. This is expected since the larger $P_{B}$, the lower are the chances of finding contiguous idle channels. Finally, Fig. 17 indicates similar trends in terms of $E_{p}$ as in the case of no guard-band reuse.

\section{Conclusions}

In this paper, we proposed an opportunistic guard-bandaware channel assignment for CRNs. Our scheme improves the CRN throughput through cooperative channel assignment, taking into consideration the guard-band constraint. The proposed channel assignment mechanism reduces the number of required guard channels for a given transmission by assigning adjacent channels as much as possible to that transmission, which significantly improves spectrum efficiency and network throughput. We first formulated the joint power control and channel assignment optimization problem, with the objective of minimizing the required spectrum for a given transmission. We showed that this problem is a BLP. Because of the exponential worst-case time complexity of BLP, 


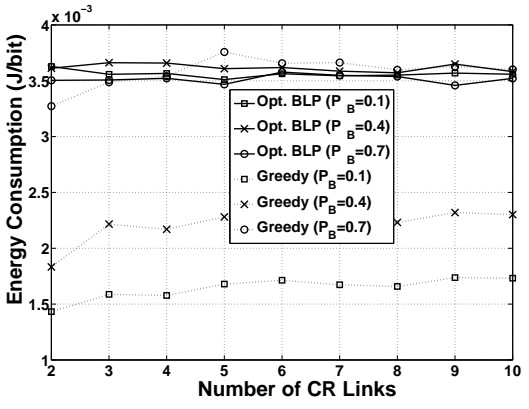

(a) $m=2$

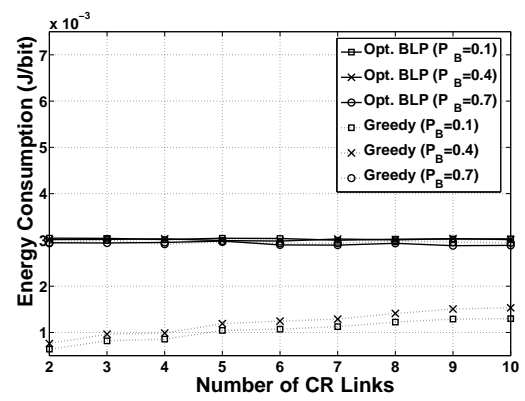

(b) $m=5$

Fig. 13. Energy consumption vs. $N$ for different values of $P_{B}$ and $m$.

we presented a near-optimal algorithm based on sequential fixing, where the binary variables are determined iteratively by solving a sequence of LPs. Based on the proposed channel assignment algorithm, we developed a CSMA/CA-based MAC protocol that enables users to dynamically utilize the spectrum. Simulation results verified the accuracy and effectiveness of the proposed protocol. We compared the performance of our protocol with that of a reference (greedy) protocol. We showed that our proposed protocol achieves up to a $180 \%$ increase in throughput over the greedy protocol, with manageable processing overhead. To the best of our knowledge, our proposed scheme is the first to account for the guardband constraint to improve the overall CRN throughput.

\section{REFERENCES}

[1] "FCC, spectrum policy task force report, ET docket no. 02-155," Nov. 2002.

[2] H. Bany Salameh and M. Krunz, "Channel access protocols for multihop opportunistic networks: Challenges and recent developments," IEEE Network-Special Issue on Networking over Multi-hop Cognitive Networks, vol. 23, no. 4, pp. 14-19, 2009.

[3] Y. Yuan, P. Bahl, R. Chandra, T. Moscibroda, and Y. Wu., "Allocating dynamic time-spectrum blocks in cognitive radio networks," in Proceedings of the ACM MobiHoc Conference, Sept. 2007.

[4] J. Jia, J. Zhang, and Q. Zhang, "Cooperative relay for cognitive radio networks," in Proceedings of the IEEE INFOCOM Conference, April 2009, pp. 794-802.

[5] A. Sabharwal, A. Khoshnevis, and E. Knightly, "Opportunistic spectral usage: Bounds and a multi-band CSMA/CA protocol," IEEE/ACM Transactions on Networking, vol. 15 , no. 3, pp. 533-545, 2007.

[6] H. Bany Salameh, M. Krunz, and O. Younis, "MAC protocol for opportunistic cognitive radio networks with soft guarantees," IEEE Transactions on Mobile Computing, vol. 8, no. 6, pp. 1339-1352, 2009.

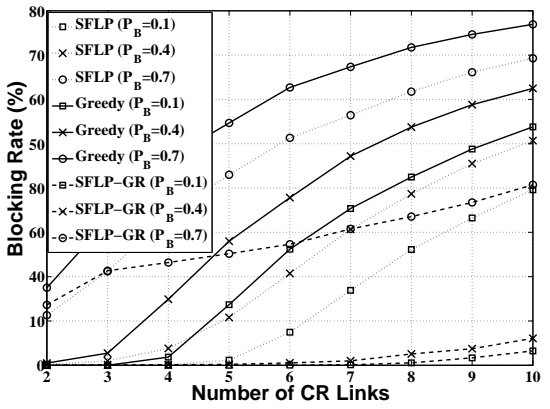

(a) $m=2$

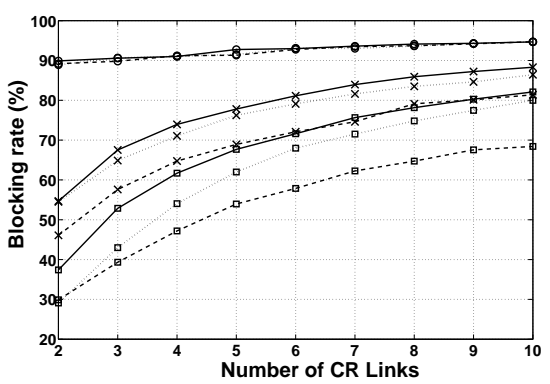

(b) $m=5$

Fig. 14. Blocking rate vs. $N$ when guard-band reuse is allowed.

[7] H. Bany Salameh, M. Krunz, and O. Younis, "Cooperative adaptive spectrum sharing in cognitive radio networks," IEEE/ACM Transactions on Networking, vol. 18, no. 4, pp. 1181-1194, 2010.

[8] A. Nasipuri and S. Das, "Performance of multi-channel ad hoc networks," International Journal of Wireless and Mobile Computing, vol. 1, no. 3/4, pp. 191-203, 2006.

[9] L. Yang, B. Zhao, and H. Zheng, "The spaces between us: Setting and maintaining boundaries in wireless spectrum access," in Proceedings of the ACM MobiCom Conference, Sept. 2010, pp. 1-12.

[10] J. Proakis, Digital Communications, McGraw Hill, 2001.

[11] J. Poston and W. Horne, "Discontiguous OFDM considerations for dynamic spectrum access in idle TV channels," in Proceedings of the IEEE DySPAN Conference, 2005, pp. 607-610.

[12] K. Nolan, P. Sutton, L. Doyle, T. Rondeau, B. Le, and C. Bostian, "Dynamic spectrum access and coexistence experiences involving two independently developed cognitive radio testbeds," in Proceedings of the IEEE DySPAN Conference, 2007, pp. 270-275.

[13] R. Chandra, R. Mahajan, T. Moscibroda, R. Raghavendra, and P. Bahl, "A case for adapting channel width in wireless networks," in Proceedings of the ACM SIGCOMM 2008 Conference, 2008, pp. 135-146.

[14] S. Joshi, P. Pawelczak, D. Cabric, and J. Villasenor, "When channel bonding is beneficial for opportunistic spectrum access networks," IEEE Transactions on Wireless Communications, vol. 11, no. 11, pp. 3942 -3956, 2012.

[15] V. Kone, L. Yang, X. Yang, B. Zhao, and H. Zheng, "On the feasibility of effective opportunistic spectrum access," in Proceedings of the 10th ACM SIGCOMM Conference on Internet Measurement (IMC'10), 2010, pp. 151-164.

[16] I. Trigui, M. Siala, and H. Boujemaa, “Optimized pulse shaping for OFDM multi-user communications over doubly dispersive channels," in Proc. of the 9th International Symposium on Signal Processing and Its Applications (ISSPA), October 2007, pp. 1-4.

[17] A. Tonello, N. Laurenti, and S. Pupolin, "Analysis of the uplink of an asynchronous multi-user DMT OFDMA system impaired by time offsets, frequency offsets, and multi-path fading," in Proceedings of IEEE Fall Vehicular Technology Conference (VTC), October 2000, pp. 1094-1099.

[18] "Second Report and Order and Memorandum Opinion and Order, ET Docket No. 04-186; FCC 08-260," 2008.

[19] J. Zhao, H. Zheng, and G.-H. Yang, "Distributed coordination in 


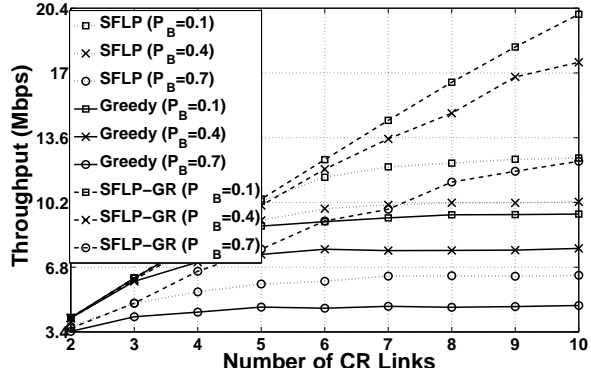

(a) $m=2$

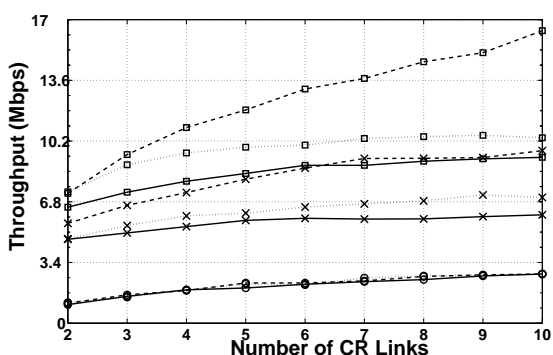

(b) $m=5$

Fig. 15. Throughput vs. $N$ when guard-band reuse is allowed.

dynamic spectrum allocation networks," in Proceedings of the IEEE DySPAN Conference, Nov. 2005, pp. 259-268.

[20] E. Arikan, "Some complexity results about packet radio networks," IEEE Transactions on Information Theory, vol. 30, no. 4, pp. 681-685, 1984.

[21] A. Behzad and I. Rubin, "Multiple access protocol for powercontrolled wireless access nets," IEEE Transactions on Mobile Computing, vol. 3, no. 4, pp. 307-316, 2004.

[22] A. Nasipuri and S. Das, "Multichannel CSMA with signal powerbased channel selection for multihop wireless networks," in Proceedings of the IEEE Vehicular Technology Conference (VTC), Sept. 2000, pp. 24-28.

[23] M. Krunz and D. Manzi, “Channel access and traffic control for dynamic-spectrum networks with single-transmit, dual-receive radios," Computer Communications, vol. 34, no. 8, pp. 935-947, 2011.

[24] L. Wolsey., Integer Programming, John Wiley \& Sons, Inc., 1998.

[25] N. Karmarkar, "A new polynomial-time algorithm for linear programming," Combinatorica, vol. 4, pp. 373-396, 1984

[26] Y. Hou, Y. Shi, and H. Sherali, "Optimal spectrum sharing for multi-hop software defined radio networks," in Proceedings of the IEEE INFOCOM Conference, May 2007, pp. 1-9.

[27] K. Bian, J. Park, and R. Chen, "A quorum-based framework for establishing control channels in dynamic spectrum access networks," in Proc. of the ACM MobiCom Conf., 2009, pp. 25-36.

[28] K. Bian and J. Park, "Asynchronous channel hopping for establishing rendezvous in cognitive radio networks," in Proc. of the IEEE INFOCOM Mini-Conf., 2011.

[29] Z. Lin, H. Liu, X. Chu, and Y. Leung, "Jump-stay based channelhopping algorithm with guaranteed rendezvous for cognitive radio networks," in Proc. of the IEEE INFOCOM Conf., April 2011, pp. 2444-2452.

[30] Y. Yuan, P. Bahl, R. Chandra, P. Chou, J. Ferrell, T. Moscibroda, S. Narlanka, and Y. Wu, "Knows: Kognitive networking over white spaces," in Proceedings of the IEEE DySPAN Conference, April 2007, pp. $416-427$.

[31] "Mesquite Software Incorporation," www.mesquite.com.

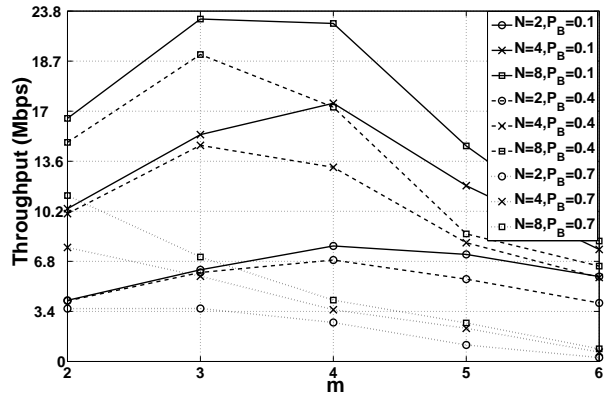

(a) Throughput vs. $m(N=2,5,8)$

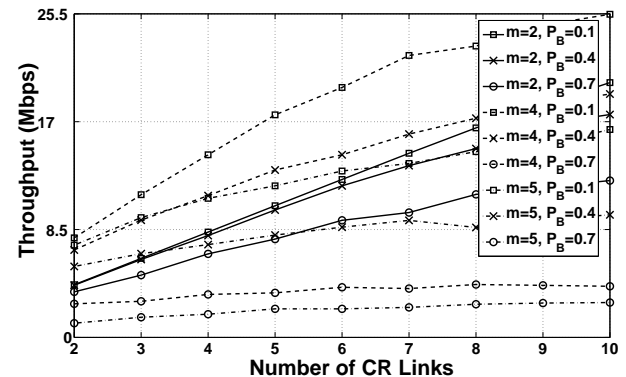

(b) Throughput vs. $N(m=2,4,5)$

Fig. 16. Throughput comparison for different values of $P_{B}$ under SFLP-GR assignment.

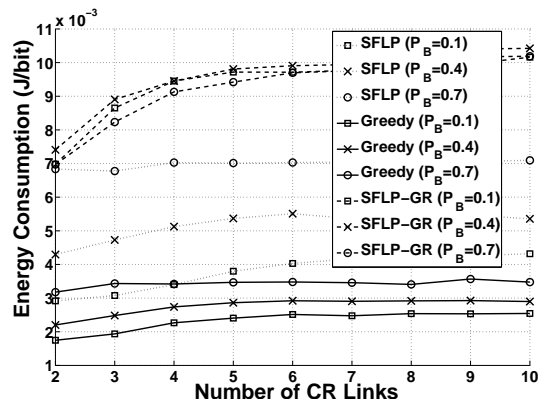

(a) $m=2$

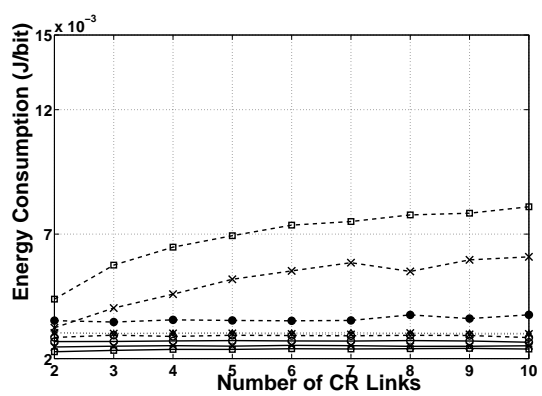

(b) $m=5$

Fig. 17. Energy consumption vs. $N$ for different values of $P_{B}$ under SFLP-GR assignment. 